\title{
Gait Phase Recognition for Exoskeleton Control USING ADAPTIVE NEURO FUZZY INFERENCE SYSTEM
}

\author{
Broniszewski Michał, Dąbroś Jakub, Iwaniec Marek, \\ Patyk Mateusz \& Wesół Jacek
}
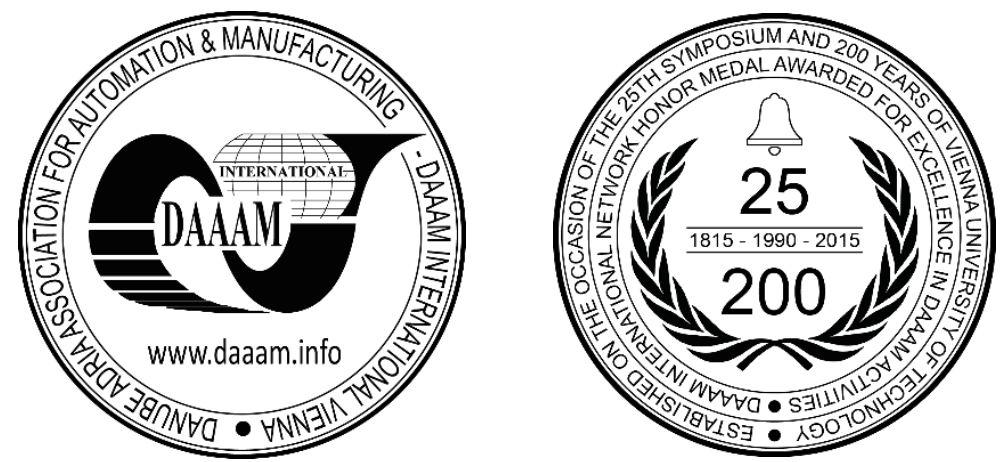

This Publication has to be referred as: Broniszewski, M[ichal]; Dabros, J[akub]; Iwaniec, M[arek]; Patyk M[ateusz], \& Wesol, J[acek] (2017). Gait Phase Recognition for Exoskeleton Control Using Adaptive Neuro Fuzzy Inference System, Proceedings of the 28th DAAAM International Symposium, pp.0845-0853, B. Katalinic (Ed.), Published by DAAAM International, ISBN 978-3-902734-11-2, ISSN 1726-9679, Vienna, Austria

DOI: $10.2507 / 28$ th.daaam.proceedings. 118

\begin{abstract}
Pressure measurement at the foot-ground contact zone provides necessary information for detecting the phases of the human gait. The human gait is a complicated process and, therefore, it is not possible to distinguish its stages simply by comparing the foot-ground surface pressure to threshold values. In this paper we propose a new method for human gait phases recognition using an adaptive network-based fuzzy inference system which processes data measured by pressure sensors located underneath footbed inserts. This method is intended to bring some additional information to the lower limb exoskeleton control system as well as for gait diagnostic purposes. The device uses only three pressure sensors for each foot. In order to verify the results of the study, measurements with sensors placed under the footbed inserts has been performed and the pressure at the foot-ground contact points during the walk has been investigated. Twelve models of gait phase classifiers were created. The performance of each classifier has been tested for a previously unknown pressure pattern that corresponds to the natural gait. Based on the quality of gait matching the test run, the best model of the classifier was selected.
\end{abstract}

Keywords: gait-phase detection; adaptive neuro fuzzy; pressure sensors; force sensitive resistor (FSR)

\section{Introduction}

Walking is an execution of repetitive sequences composed of the movements of the lower limbs in order to proceed forward with the simultaneous keeping of proper balance [1]. It is also one of the most convenient forms of locomotion [1] which enables people to perform basic daily activities [2]. A single, non-recurring sequence of movement performed by one leg is called a gait cycle [1]. Gait cycle considered in the extended perspective can be divided into eight unique walking phases [3].

Recognition of user current walking phase is a key element of the control algorithms of the exoskeleton of the lower extremities [3][4][5]. Very often, in order to simplify the control system in these devices, designers reduce the amount of reproduced walking phases, in spite of the awareness of seven or more gait phases [3][4][5]. 
In most cases, signals such as threshold values of pressure sensors, angles observed in knee and hip joints, or readings from electromyographic sensors, are used to determine the state of the structure and its operator [3][4][6].

While the angular trajectories should be very similar for most people, the ground reaction forces (GRF) will vary according to the weight of the tested person. For this reason, we believe that gait phase recognition based on the input value comparison with a set of predetermined threshold values is an inadequate and strongly restrictive approach to the functionality of an exoskeleton.

Modeling systems based on traditional mathematical tools is not suitable for working with vaguely formulated or difficult to define models that are better suited for use with fuzzy reasoning systems simulating human decision-making [7].

In this paper, we propose a solution to the problem of gait phase detection for diagnosis or use in the control system of the lower limb exoskeleton in form of adaptive neuro-fuzzy inference systems. The proposed adaptive system is based on an artificial neural network, in which neurons are defined and linked together in such a way as to simulate a fuzzy reasoning system [7]. The great advantage of this solution is that there is no need to know the exact model of the object or process being analyzed. In order to determine the appropriate number of fuzzy sets to which the reading at any given time may belong, it is sufficient to provide input data and targeted output data with an addition of an appropriate input data conceptual analysis.

\section{Review}

Recent advances in a wearables have contributed to the development of techniques for a gait phase automatic detection and recognition [8]. These techniques are based on the measurement of muscle activity (EMG) [9], joint angles by using encoders or goniometers [10][11], but also accelerometers and gyroscopes [12] or the fusion of them both [13].

Walking aids such as exoskeletons and active orthoses use built-in sensors to control and to detect a user's intentions [4][9]. However, methods based on EMG will not necessarily be the best solution for supporting devices due to their individual variability [2]. There are also problems with electrodes placement on the body and difficulties with EMG signal processing. Exoskeleton constructions frequently use encoders or potentiometers for measuring joint angles, but also inertial measurement units (IMUs) as well as pressure sensors [14].

Foot-to-ground contact detection signals are directly translated into information about the current walking phase, hence the common use of pressure sensors in exoskeleton construction [HAL]. However, GRF signals do not provide information for the device control but give the basis for human movement detection which allows the control systems to adjust the control algorithms to the current walking phase [2]. However, it is often a problem to adapt pressure sensor systems to accommodate to different footprints and working conditions [14].

Research shows that the use of artificial neural networks based on force sensitive resistors (FSRs) located under the foot are the most precise methods of gait phase recognition, even for cases where the network has not been taught before. Similar neural network, which instead used accelerometer data, achieved significantly higher error values calculated with respect to gait pattern. Additionally, the accelerometers introduced recognition delays of over $120 \mathrm{~ms}[15]$.

Much previous work uses at least four FSR sensors in their systems, which are typically located in four distinct places: under the heel, 1st, 4th or 5th metatarsal bones, and under the hallux [2][13][15]. Jung et. al. in their work, used eight pressure sensors located between the upper and lower shoe inserts [14]. Thanks to the use of velcro materials, the position of each sensor can be changed at any time to match the size of the measurement system to different foot sizes. This approach requires that the location of sensors individually adjusted for every patient before use. The commonly used method for determining gait phases is a method that compares readings with predetermined threshold values. The method is called the threshold method or the discrete event method [2]. Jung et. al. use the collected data from all eight sensors of the exoskeleton foot to determine the stance and swing phase. If the data from each sensor exceeds the predetermined threshold, it means that the exoskeleton is in the stance phase [15]. The use of eight pressure sensors to determine two gait phases is definitely uneconomical, as such a system can be replaced with one sensor on each foot.

Significantly better results are obtained by using artificial neural networks using pressure sensor or accelerometer data [15]. The authors used four pressure sensors that became inputs for the multi-layer perceptron (MLP). The structure of the used network consisted of four neurons (for each input), one output neuron and fifteen neurons in each of the two hidden layers. The network was taught to detect the stance phase and swing phase. Its results showed a 2.8\% error [15]. Despite the high efficiency of the method, it was possible to recognize only two phases of walking, which in the case of the support device control is insufficient.

The use of gait phases in control algorithms requires the changes between each phase to be more fluid rather than rapid. Similarly, during the walk, walking phases are very smooth, since the human gait is extremely energy efficient and to a large extent, allows suppression of the reaction forces of the substrate [1]. The smooth transitions between the walking phases were obtained by Kong [2], who used the fuzzy logic method to recognize the walking phases using four pressure sensors placed under the shoe insert. The approach proposed by the author allowed the detection of six phases of walking [16]. However, the use of fuzzy logic requires an understanding of the problem to formulate a rule base [2]. Our adaptive neuro-fuzzy inference system (ANFIS), unlike fuzzy models, does not require previous knowledge of the object being analyzed and does not require the creation of a rule base. The rule base is created by the system based on the input data provided, thus excluding the possibility of error. 


\section{Materials and methods}

The simplest walk cycle can be divided into two stages: stance (or support) and swing [1]. In the extended view of the gait cycle, it has been divided into eight unique phases [1]. Each phase has its own footprint pattern, that is, the surface that contacts the ground. The distinction between the stance and swing period is possible with one pressure sensor on each foot (pressure is present, or no pressure is present). On the other hand, the distinction between all eight walking phases requires the use of multiple measuring systems.

\subsection{Design concept}

Human gait is an extremely complex operation, so it is difficult to determine when the gait cycle begins and ends. The Rancho Los Amigos Committee from the American National Rehabilitation Center has established that the heel strike (Initial Contact, IC) phase is the initiating phase of the gait cycle [1]. Contrary to this theoretical consideration, this study considered a first phase of the gait (zero index) to be the swing phase, secondary to the heel strike and the following phases.

During walking, body mass swings across the arch along the surface of the foot from the heel toward the fourth and fifth metatarsal bones, and then follows a straight line toward the large toe. Body weight is transferred through the closed kinematic chain created by the skeleton to the ground. The places of greatest pressure at the foot-ground contact zone correspond to the anatomical structure of the skeleton of the foot [17]. Although tissues act as depreciation, the greatest pressure values occur at the areas where the foot bones are closest to the ground. Therefore, it is reasonable to place the pressure sensors in these places.

In the presented solution, only three pressure sensors were used for each foot. Sensors were located at the locations of the largest reaction forces of the substrate. Fig. 1 shows the location of the sensors placed under the footbed inserts. The first sensor is located at the center of the heel (Fig 1, label 3), the second is located near the fifth metatarsal bone (Fig. 1, label 2), and the third is placed under the hallux (Fig. 1 label 3).

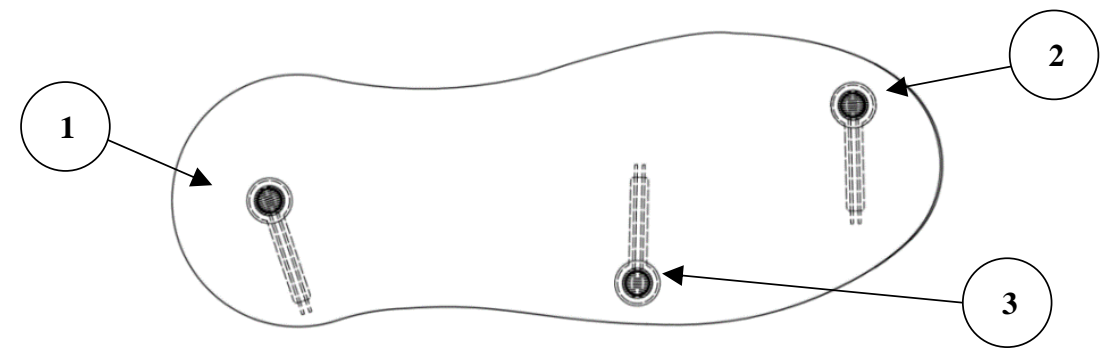

Fig. 1. Location of the pressure sensors: 1 - heel, 2 - 5th metatarsal bone, 3 - hallux.

Each gait phase can be identified by a unique support surface. Fig. 2a shows the sequence of changes in the foot support surface that contacts the ground during the support period. The Initial Contact (IC) phase begins with the first contact of the heel with the ground. It takes a very short time (about $2 \%$ of the total gait cycle, GC), so it is often analyzed along with the Loading Response (LR) phase [1].

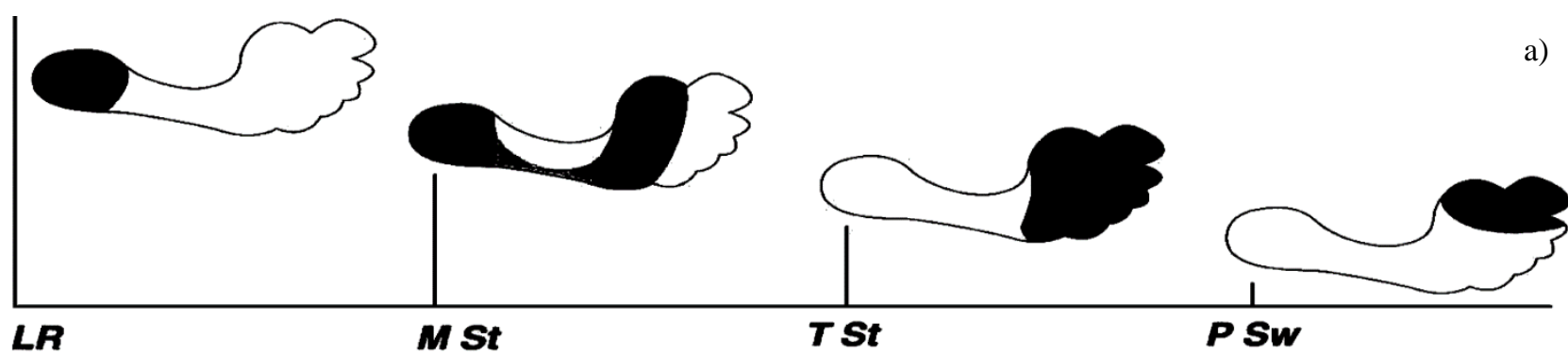

Fig. 2a. Sequence of changes in the foot support areas during stance. Based on [18].

b)

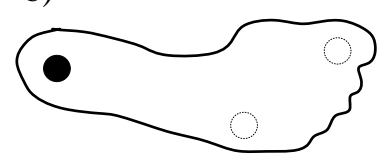

c)

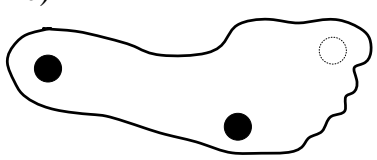

d)

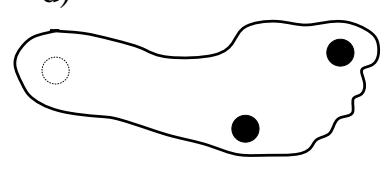

e)

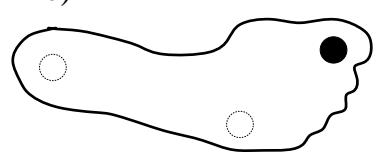

Fig. 2b-e. Representation of pressure exerted by the foot on the sensors at specific gait phases. 
At this time only the back of the foot, specifically the heel, contacts the ground, while a body weight is gradually transferred to the lower limb. During the initial two phases of the walk, a large amount of pressure is recorded from the sensor located on the heel. Fig. $2 b$ shows the pressure measured by the sensor, which has been represented by the black circle. The other two sensors record no pressure or very small pressure, as shown in Fig. $2 b$ - circles in short dash lines. During Mid Stance (M St) phase, the foot remains straight, so pressure is measured on the heel and the fifth metatarsal bone (Fig. 2c). During Terminal Stance (T St) phase, the heel pressure disappears while the pressure on the forefoot increases. At this time pressures are recorded by sensors located under the fifth metatarsal bone and under the hallux (Fig. $2 \mathrm{~d})$. The last recordable phase during the support period is the Pre-Swing ( $P S w)$ phase, in which the pressure is only recorded by the sensor placed under the hallux (Fig. 2e).

During the swing period, the foot does not touch the ground, so each sensor on the transferred leg will measure the pressure close to zero. Therefore, the swing phases will be interpreted as one phase.

Despite the fact that only three sensors were used, all of the subphases during the stance period could have been distinguished. Sensor placement points were selected based on analysis of the changes in the foot support areas during stance (Fig. 2a). Placing sensors under the heel and hallux is necessary. On the other hand, placing the sensor under the fifth metatarsal bone allows the distinction between the Terminal Stance phase and the Pre-Swing phase. Such sensor placement ensures that each phase is uniquely distinguished on the basis of a readout from at least one sensor.

\subsection{Hardware, software and data acquisition}

The measuring system consists of three pressure sensors, resistance-voltage converters, voltage followers and microcontroller with analogue-to-digital converters (Fig. 3). The acquisition system was based on an Arduino Due developer kit with an Atmel ATSAM3X8E processor (84 MHz, 32 bit ARM Cortex-M3 architecture). The microcontroller is equipped with 12-bit analog-to-digital converters, resulting in measurement accuracy of $\approx 0.8 \mathrm{mV}$ with a measuring range of $3.3 \mathrm{~V}$.

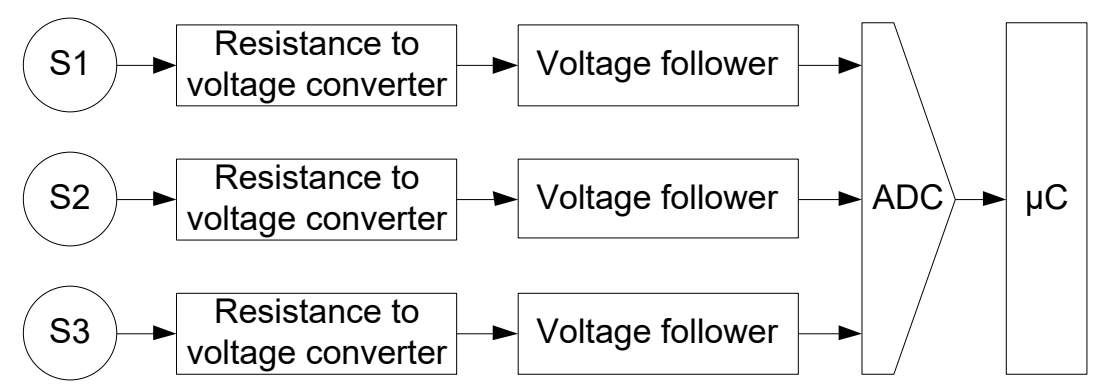

Fig. 3. Block diagram of the measuring system.

Three CP151NS sensors from IEE International Electronics \& Engineering were used for one foot. The used sensors are the Force Sensitive Resistor (FSR), which shows a decrease in resistance due to the increasing pressure exerted on them. Each of these sensors is plugged into a voltage divider that comes with the potentiometer. It allows the voltage range to be adjusted at the output of the $V_{O U T}$ divider (1) by controlling the resistance of the $R_{P O T}$ potentiometer.

$$
V_{\text {OUT }}=V_{+} \frac{R_{P O T}}{R_{P O T}+R_{F S R}}
$$

In such an arrangement, the voltage at the output of the divider increases as the force applied to the sensor surface increases, which is equivalent to the decrease in the resistance of the $R_{F S R}$ sensor. Potentiometer resistance $R_{P O T}$ was set to value $10 \mathrm{k} \Omega$ and $3.3 \mathrm{~V}$ supply voltage $V_{+}$was applied to the voltage dividers. The output of each voltage divider was connected to the voltage follower input and then the output of the divider was connected to the corresponding ADC input.

The transmission and pressure sensors program was written in $\mathrm{C}++$ using Atmel Studio 7, then compiled using Arduino toolchain and finally loaded into the microcontroller memory.

The subject whose gait data was collected was $185 \mathrm{~cm}$ tall and weighed $77 \mathrm{~kg}$. The measurements were made for three sensors located under the footbed of the right foot. The sensors have been positioned as shown in Fig. 1. The recorded data was sampled with $1.3 \mathrm{kHz}$ sampling rate, filtered in real time with a digital lowpass filter (2) implemented in the program structure and sent to a PC via a serial port.

$$
y[i]=\alpha * x[i]+(1-\alpha) * y[i-1]
$$

$y[i], x[i]$ and $x[i-1]$ denote the filter output in $i$-th sample respectively, the output from the previous sample filter, and the current readout value from the ADC. Parameter $\alpha$ is a smoothing agent and is in the range $(0,1)$, where the value close to zero means a strong smoothing of the waveform. The value of $\alpha$ was set at 0.1 . 


\subsection{ANFIS}

Angular variations in the joints of a subject's lower limbs during a walk in a given cycle can be described as nonlinear in time [1]. Taking into account the differences between individuals, such as varying body weight or foot geometry or the possibility of deformation during the development of anatomical arches of the foot (e.g. flat foot), it is impossible to clearly describe the human gait with an exact mathematical model that can be applied to population at large. Pressure distributions in feet with lowered physiological arches due to the transfer of part of the human weight to a healthy support area that is not available in healthy people foot will appear to be completely different than those with properly developed foot geometry. It is therefore difficult to create an accurate, commonly used, gait classifier based on standardized sizes, in which case they are usually affiliated to collections limited by the predefined thresholds. This requires the use of membership to collections defined by the use of traditional logic used by exoskeleton creators. For example, if the detection of the passage between the heel strike and the loading response is defined as exceeding the threshold pressure value by the sensor readings on the fifth metatarsal bone, it is essential to provide information on the body weight of the threshold value used. If the calculated hypothetical value is assumed to be equal to half of the measuring range, based on the pressure-to-weight ratio of the $50 \mathrm{~kg}$ person-to-weight contact, the $100 \mathrm{~kg}$ subject will exceed the threshold value at an earlier stage of analogically considered walking cycle [20]. In addition, surface contact patterns for different patients may be different, hence threshold-based methods may not be able to correctly interpret the current walking phase in such diverse cases [2]. Due to the strong nonlinearity of a human gait dynamics in exoskeleton control systems, the control models vary depending on the phase detected at any given moment [4][14]. Despite problems with the manufacture of systems suitable for different footprints and conditions, classifiers based on pressure sensors are commonly used in active lower leg orthoses [14].

The proposed solution to the above problems is the adaptive neural-fuzzy inference system (ANFIS), first described by Jang with his example of systems that allow the modeling of nonlinear functions [7]. The ANFIS structure is based on an artificial neural network and consists of five layers in which the neurons have different structures and perform different functions [19]. The proposed structure is designed to respond to the analyzed problem. Each layer corresponds to another element of the fuzzy inference system. For the purpose of explaining the principle of operation of the network, a model based on three fuzzy sets describing each of the inputs was adopted.

The first, in order of the signal flow direction, is the input layer with the number of $n=3$ perceptrons carrying information about the filtered data collected by the pressure sensors. As to the values, the individual nodes correspond to the values of the sensors on the heel, the fifth of a metatarsal, and the toe. The second layer present in the model is the layer of the input membership functions. At this level of the algorithm, each of the inputs is described by $m=$ 3 membership functions, symbolizing respectively: no pressure or low pressure, medium pressure, high or total pressure. In the case of the second set we cannot speak of the mean as the value describing the signal, but as the subjective conclusion of the network. The value stored by each node in this layer is the result calculated by substituting the neuron response of the input layer with the pattern for the applied membership function. The third layer is the layer of inference rules, which contains $m^{n}=3^{3}=27$ rules describing the inference system with logical expressions. The scheme of operation of this network layer can be presented in linguistic form "if $\mu_{0, i}$ and $\mu_{1, j}$ and $\mu_{2, k}$ then $\mu_{o u t}$ ", or in mathematical form (3), where $\mu_{0, i}, \mu_{1, j}$ and $\mu_{2, k}$, are the results of the input membership functions, where the indices $\{i, j, k\}$ tell us which of the sets is considered for each input, as $\mu_{\text {out }}$ analogous value for the layer output.

$$
\mu_{0, i} \wedge \mu_{1, j} \wedge \mu_{2, k} \Rightarrow \mu_{\text {out }}
$$

The penultimate in the model, the layer of the function of the membership of the outputs is composed of the same number of fuzzy sets as the number of rules in the preceding layer $m^{n}=3^{3}=27$. The values received by each neuron are subjected to the input of membership function of the layer output and they are dependent directly on the inference of the previous layer. This layer's nodes' output values have been normalized. The output value of the model is provided by the layer of aggregation and defuzzyfication. It is a layer composed of only one neuron with $m^{n}=3^{3}=27$ inputs, each of a specific weight, which, when collected and defuzzyfied, is represented as a specific crisp output value.

The parameters of the model that can be changed (apart from the number of inputs already mentioned and the number of fuzzy sets belonging to the given input) include: the shapes of the membership functions, the choice between the AND and OR functions and the corresponding $t$-norm implemented in the rules base layer, learning vectors, $t$-norm for implication, output aggregation definition, and output defuzzification method. Our study involved the preparation and teaching of a set of 12 networks, two of which served as "master" networks, the starting point for modifying the parameters, which differed in learning time between them. These are networks c) and d), with the learning of the first being interrupted in about $80 \%$ of the second learning period. For a detailed description of the variability of the models, see Table 1.

\section{Results}

Neural network learning results were derived from a mean squared error between the pattern and the neural network result and presented in Table 1. The highest of the errors noted was the model with the linear function of the outputs membership and it was an error of 2 orders of magnitude larger than the others. 
On the other hand, the smallest error characterized the network with an increased number of fuzzy sets - 5 for each input. The standard parameters for the study were: 19991 samples in teaching vectors, Gaussian input functions, the function of membership of outputs in the form of one-element sets, using the t-standard operation in the form of the product of sets, and finally using the method of teaching with the use of reverse propagation error.

\begin{tabular}{|c|c|c|c|c|c|c|c|}
\hline Model & $\begin{array}{l}\text { Length of } \\
\text { learning } \\
\text { matrix } \\
\text { [samples] }\end{array}$ & $\begin{array}{c}\text { Fuzzy sets } \\
\text { number }\end{array}$ & $\begin{array}{c}\text { Input } \\
\text { membership } \\
\text { function }\end{array}$ & $\begin{array}{l}\text { Output membership } \\
\text { function }\end{array}$ & $\begin{array}{c}\text { t-norm } \\
\text { operation } \\
\text { for AND }\end{array}$ & $\begin{array}{c}\text { Network learning } \\
\text { method }\end{array}$ & $\begin{array}{l}\text { Network } \\
\text { Error }\end{array}$ \\
\hline a) & $\underline{10000}$ & {$\left[\begin{array}{lll}3 & 3 & 3\end{array}\right]$} & Gauss & singletons & prod & back propagation & 0,2700 \\
\hline b) & $\underline{15000}$ & {$\left[\begin{array}{lll}3 & 3 & 3\end{array}\right]$} & Gauss & singletons & prod & back propagation & 0,2740 \\
\hline c) & 19991 & {$\left[\begin{array}{lll}3 & 3 & 3\end{array}\right]$} & Gauss & singletons & prod & back propagation & 0,2875 \\
\hline d) & 19991 & {$\left[\begin{array}{lll}3 & 3 & 3\end{array}\right]$} & Gauss & singletons & prod & back propagation & 0,2803 \\
\hline e) & 19991 & {$\left[\begin{array}{lll}3 & 3 & 3\end{array}\right]$} & triangular & singletons & prod & back propagation & 0,2688 \\
\hline f) & 19991 & {$\left[\begin{array}{lll}3 & 3 & 3\end{array}\right]$} & trapezoid & singletons & prod & back propagation & 0,2989 \\
\hline g) & 19991 & {$\left[\begin{array}{lll}3 & 3 & 3\end{array}\right]$} & Gauss & linear & prod & back propagation & 12,0755 \\
\hline h) & 19991 & {$\left[\begin{array}{lll}3 & 3 & 3\end{array}\right]$} & Gauss & singletons & $\underline{\min }$ & back propagation & 0,3040 \\
\hline i) & 19991 & {$\left[\begin{array}{lll}3 & 3 & 3\end{array}\right]$} & Gauss & singletons & prod & hybrid & 0,2649 \\
\hline j) & 19991 & {$[444]$} & Gauss & singletons & prod & back propagation & 0,2759 \\
\hline k) & 19991 & {$\left[\begin{array}{lll}5 & 5 & 5\end{array}\right.$} & Gauss & singletons & prod & back propagation & 0,2460 \\
\hline 1) & 19991 & {$[777]$} & Gauss & singletons & prod & back propagation & 0,5992 \\
\hline
\end{tabular}

Table 1. Examined models of gait classifiers. The column representing the number of fuzzy sets presents the vector of the number of sets for each input.

Fig. 4 shows the comparison of the determined gait phase obtained from the operation of a given model with gait phase patterns, which were manually defined in the same way (using the same phase separation criteria) as for generating the resultant pattern vectors for the data provided to the learning network.

Waveform observation allows to provides a reference for the reliability of the numerical results describing the network's mean squared error. Single samples with high error values relative to the corresponding standard samples may lead to an increase in RMSE value. Detecting and counteracting such phenomena can lead to obtaining better classifiers than would be suggested by learning outcomes.

In addition to the network comparison chart, the pattern of absolute error values (Fig. 5) was also determined, allowing to confront the network operation for each phase of the walk. The highest values of errors were observed at the end of each walk cycle (assuming the order of gait phases used in the study). The shape of gait pattern should be examined carefully. The model is characterized by low error rates and relatively small derivatives, but it contains single peaks with higher values, which can be considered as a good match for the network.

This means that for most of the walk cycle, the fit to the pattern was high, but there were moments when the gait phase obtained from the classifier differed significantly from the one observed in reality. On the other hand, patterns characterized by multiple local maxima or relatively high values with minor variations may be understood as inferior network matching and greater inconsistency in real and classified walking phases.

Fig. 6 shows a direct comparison of the mean error of the classifier's performance against the standard shown in the average error and maximum error. The top line shows a comparison of all the discussed models, but due to significant differences ( 2 orders of magnitude) between the models, it was decided to reject two of them and display the crop data again in the following line, zeroing values for skipped models. The smallest RMSE model with five fuzzy sets for each of the inputs did not turn out to also be the model with the lowest maximum error, which was characterized by model e) from Table 1 which was using the triangular function of the inputs.

On both Fig. 4 and Fig. 5, only portions of the whole 40 seconds duration measurement are visualized. Fig. 6 shows the results from the analysis of the whole chart. For this reason, the maximum error values may not be visible in previous waveforms. 

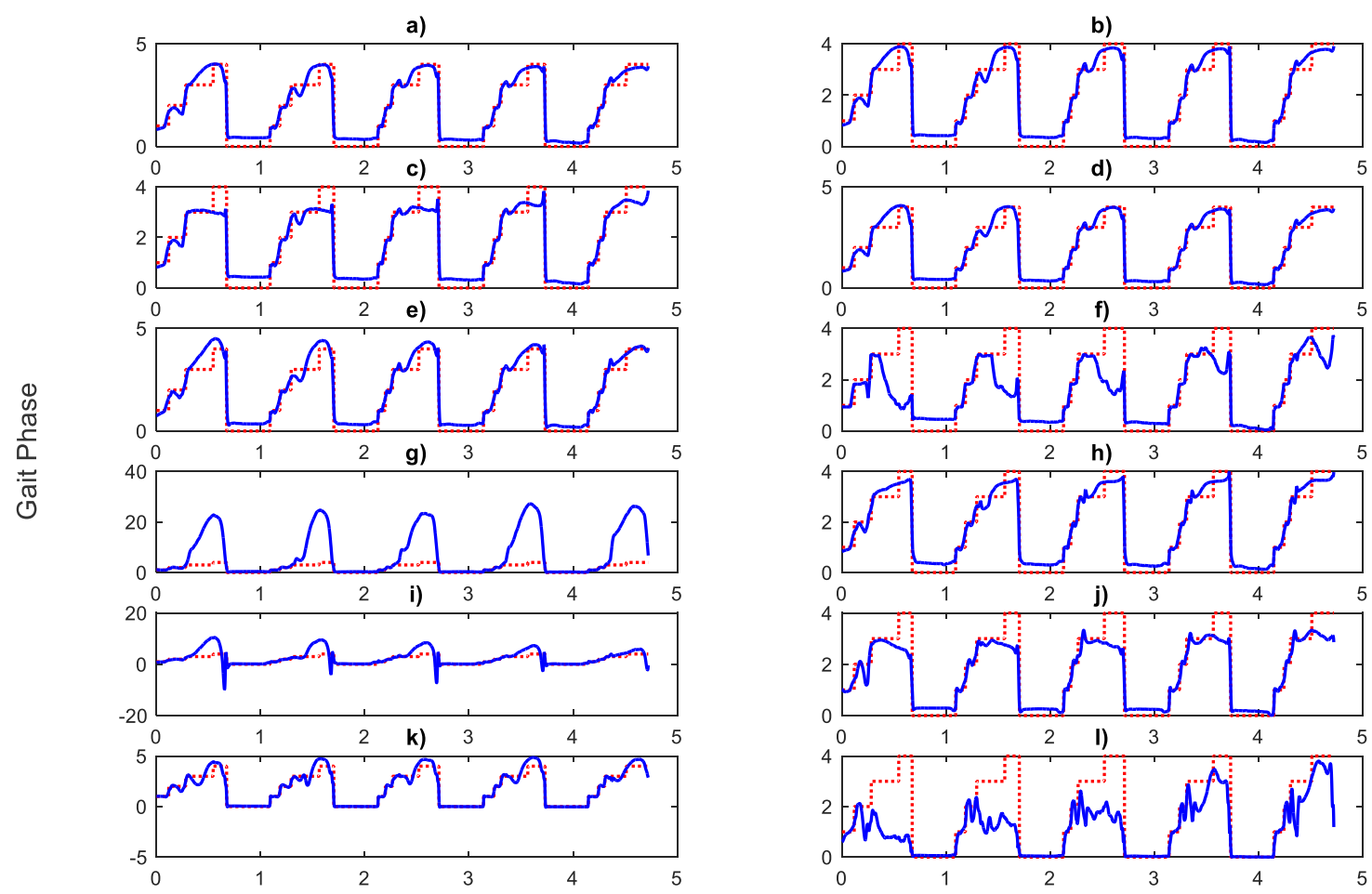

Time $[\mathbf{s}]$

Fig. 4. Comparison of ANFIS results with manually prepared pattern. Only a fragment of the entire chart is presented. The individual graphs are described in the following lines in Table 1 showing the parameters of the system.
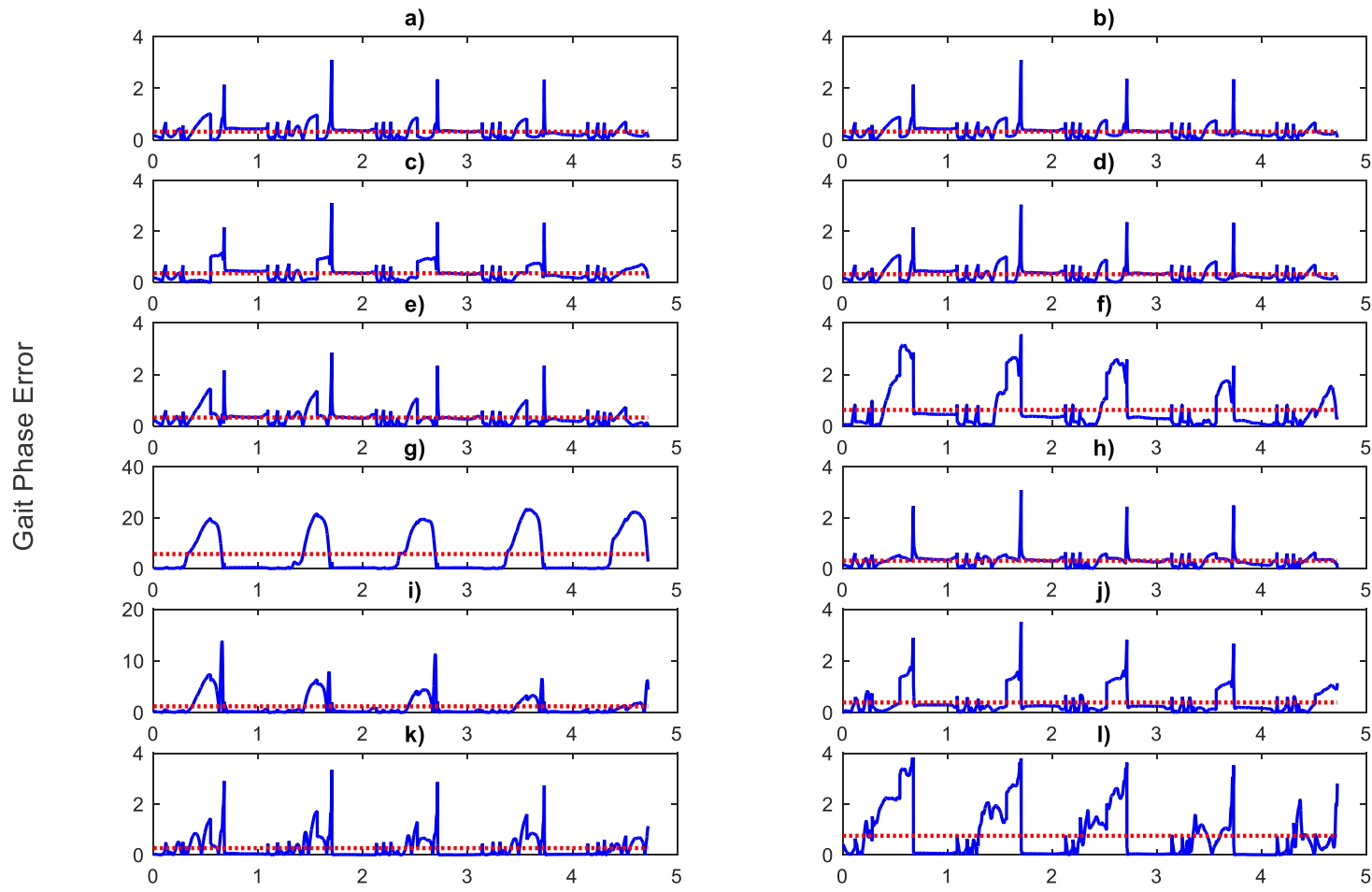

Time [s]

Fig. 5. Comparison of absolute error values of ANFIS results. Only a fragment of the entire waveform is presented. The dashed line symbolizes the average error value of the model. The individual graphs are described in the following lines in Table 1 showing the parameters of the system. 

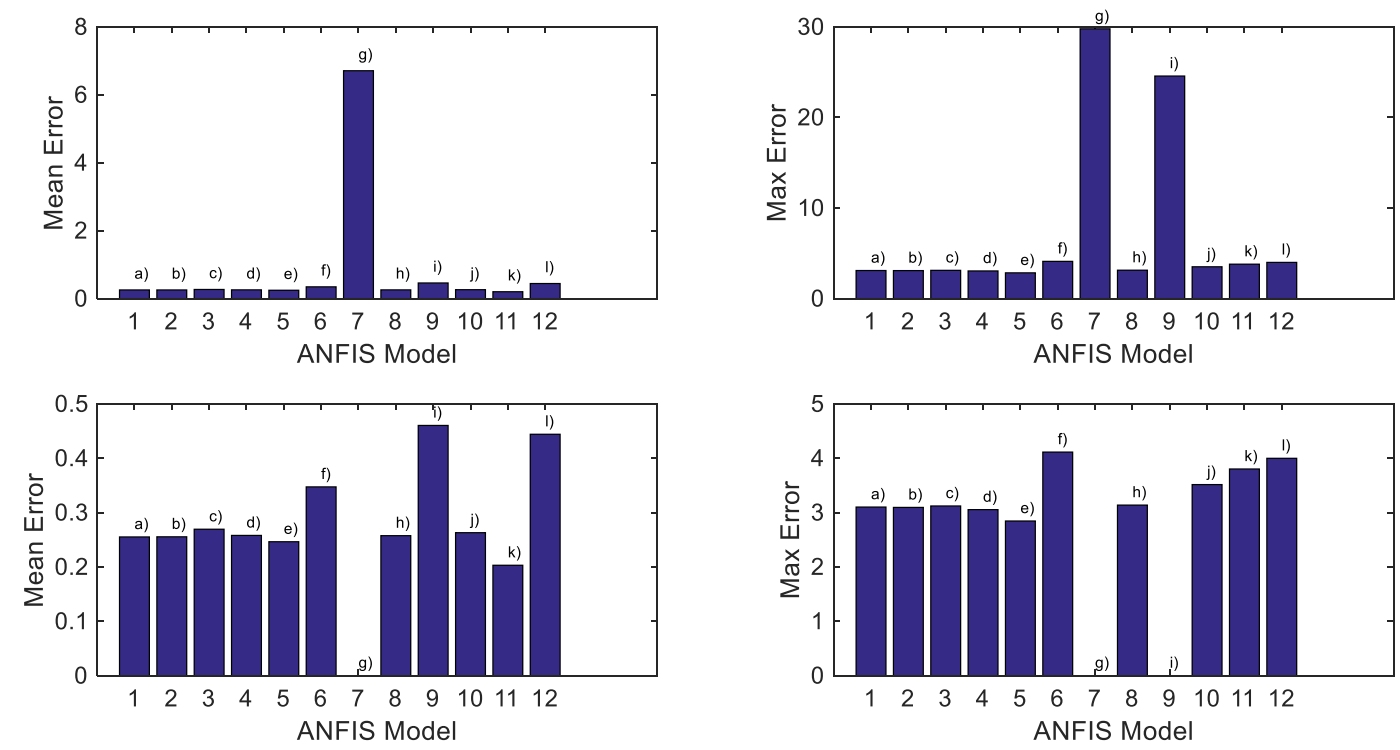

Fig. 6. Comparison of mean and maximum errors for each model. The top row contains graphs showing all tested models. The bottom line excludes results for models with much higher error values than the rest of the models.

\section{Conclusion}

This article presents a proposal for classifiers based on adaptive neuro-fuzzy inference systems that enable gait phase recognition. Proposed neural network was designed for use in control systems of bipedal robots including lower limb exoskeletons for rehabilitation or enhancement of physical capabilities of the operator.

The characteristic feature of the proposed classifiers is the use of only three inputs corresponding to the pressure sensors mounted at the foot-ground contact point, which considerably reduces the number of operations required to be performed by the central processing unit of the control system. This solution provides much shorter response time of the system, thereby allowing better control of the device stability, which increases the safety of the exoskeleton operator.

Twelve classifier models differing in their parameters were tested. The best results were achieved by model which was provided in full trajectories as learning vectors with a structure of five fuzzy sets, each described by the Gaussian membership function in sets. Outputs of the mentioned function were described by the membership function to oneelement sets which used the $t$-norm operation in form of the product of fuzzy sets and finally using the method of output defuzzification using a weighted average. Despite higher than other models' values of maximum errors, the overall alignment of the classifier's output pattern to the input pattern was the best, and with proper data manipulation, it should lead to ultra-precise classifiers with low specificity, allowing work with a wide range of people.

Despite theoretically greater possibilities to differentiate the input states for the seven fuzzy sets model, it turned out that the results were unsatisfactory. Improvement of performance against classifiers using less fuzzy sets was expected. It is concluded that the low value of membership for a sample value centered between the center of gravity of two adjacent sets is more advantageous to the system than the ability to qualify a given sample to an additional set. This is a desired feature for implementation on microprocessor systems - fewer sets mean less demand for computing power. It would be useful, however, to investigate the reproducibility of the mentioned feature using different network parameters.

Further work should focus on carrying out measurements on a larger group of subjects with varying body weight. It is also proposed to research a problem of handicapped patients with walking difficulties in order to assess the ANFIS use for gait diagnostics.

\section{References}

[1] Perry, J. (1992). Gait analysis: normal and pathological function, SLACK, 978-1-55642-192-1, Thorofare, NJ

[2] Kong, K. \& Tomizuka, M. (2008). Smooth and continuous human gait phase detection based on foot pressure patterns, 2008 IEEE International Conference on Robotics and Automation, Pasadena, CA

[3] Vaughan, C. L.; Davis, B. L. \& O'connor, J. C. (1992). Dynamics of human gait, Human Kinetics Publishers, 9780-620-23558-6, Champaign, Illinois

[4] Kazerooni, H.; Racine, J. L.; Huang, L. \& Steger, R. (2005). On the Control of the Berkeley Lower Extremity Exoskeleton (BLEEX), Proceedings of the 2005 IEEE International Conference on Robotics and Automation, Barcelona, Spain, pp. 4353-4360, doi: 10.1109/ROBOT.2005.1570790

[5] Herr H. et al. Exoskeleton for running and walking, U.S. Patent No. 2007/0123997 A1, May 31, 2007

[6] Kenta, S.; Gouji, M.; Hiroaki, K.; Yasuhisa, H. \& Yoshiyuki, S. (2007). Intention-based walking support for paraplegia patients with Robot Suit HAL, Advanced Robotics, Vol. 21, No. 12, pp. 1441-1469 
[7] Jang, J.-S. R. (1993). ANFIS: Adaptive-Network-Based Fuzzy Inference System, IEEE Transactions on Systems, Man, and Cybernetics, Vol. 23, No. 3, pp. 665-685

[8] Tao, W.; Liu, T.; Zheng, R. \& Feng, H. (2012). Gait analysis using wearable sensors, Sensors, Vol. 12, No. 2, 2012, pp. 2255-2283

[9] Sankai, Y. (2010). HAL: Hybrid assistive limb based on cybernics, Robotics Research, Springer, 2010, pp. 25-34

[10] Zoss, A. B.; Kazerooni, H. \& Chu, A. (2006). Biomechanical design of the Berkeley lower extremity exoskeleton (BLEEX). IEEE/ASME Transactions On Mechatronics, Vol. 11, No. 2, pp. 128-138.

[11] Donno, M.; Palange, E.; Di Nicola, F.; Bucci, G. \& Ciancetta, F. (2008). A new flexible optical fiber goniometer for dynamic angular measurements: Application to human joint movement monitoring. IEEE Transactions on Instrumentation and Measurement, Vol. 57, No. 8, 2008, pp. 1614-1620

[12] Zeng, H, \& Zhao, Y. (2011). Sensing movement: Microsensors for body motion measurement, Sensors, Vol. 11, No. 1, 2011, pp. 638-660

[13] Parikesit, E.; Mengko, T. L. R. \& Zakaria, H. (2011). Wearable gait measurement system based on accelerometer and pressure sensor, Proceedings of 2011 2nd International Conference on Instrumentation, Communications, Information Technology, and Biomedical Engineering (ICICI-BME), Bandung, Indonesia, pp. 395-398

[14] Mijailovic, N.; Gavrilovic, M.; Rafajlovic, S.; Đuric-Jovicic, M. \& Popovic, D. (2009). Gait phases recognition from accelerations and ground reaction forces: Application of neural networks. Telfor Journal, Vol. 1, No. 1, pp. 34-36

[15] Jung, J. Y.; Heo, W.; Yang, H. \& Park, H. (2015). A neural network-based gait phase classification method using sensors equipped on lower limb exoskeleton robots. Sensors, Vol. 15, No. 11, pp. 27738-27759

[16] Kong, K. \& Tomizuka, M. (2009). A gait monitoring system based on air pressure sensors embedded in a shoe. IEEE/ASME Transactions on mechatronics, Vol. 14, No. 3, pp. 358-370

[17] Cavanagh, P. R. \& Michiyoshi Ae. (1980). A technique for the display of pressure distributions beneath the foot. Journal of biomechanics, Vol. 13, No. 2, pp.69-75

[18] Barnett, C. H. (1956). The phases of human gait. The Lancet, Vol. 268, No. 6943, pp. 617-621

[19] Nyrkov, A.; Chernyi, S.; Zhilenkov, A. \& Sokolov, S. (2016). The use of Fuzzy Neural Structures to Increase the Reliability of Drilling Platforms, Proceedings of the 26th DAAAM International Symposium, pp. 0672-0677, B. Katalinic (Ed.), Published by DAAAM International, 978- 3-902734-07-5, 1726-9679, Vienna, Austria, DOI: 10.2507/26th.daaam.proceedings.091

[20] Birtane, M. et al. The evaluation of plantar pressure distribution in obese and non-obese adults, Clinical Biomechanics, Vol. 19, No. 10, pp. 1055 - 1059 